\title{
New Insights into the Role of ENPP1 in Insulin Resistance
}

\section{Wentong Pan ${ }^{1}$, Manisha Chandalia ${ }^{1}$ and Nicola Abate ${ }^{1 *}$}

\section{Keywords: ENPP1; ENPP1 K121Q; Obesity; Insulin Resistance}

Ectonucleotide pyrophosphatase/phosphodiesterase 1 (ENPP1) is a transmembrane glycoprotein highly expressed in adipocytes and also found in other tissues involved in glucose and lipid metabolism, including liver, skeletal muscle and beta cell of the pancreas. Early studies by Maddux and Goldfine [1] demonstrated that ENPP1 interacts with the insulin receptor at the level of the alpha subunit. This interaction results in decreased insulin-mediated activation of the tyrosine phosphorylation in the beta-subunit of the insulin receptor, and downstream insulin signaling activation. As a consequence, increased expression of ENPP1 in cell cultures has been shown to induce cellular insulin resistance [2]. The interest in this mechanism of cellular insulin resistance is accentuated by reports of an effect of ENPP1 gene variant on susceptibility to systemic insulin resistance, type 2 diabetes and cardiovascular disease [3]. Several investigators have pointed out that a common missense single nucleotide polymorphism of the ENPP1, the K121Q, associates with type 2 diabetes in humans [4-12]. The goal of this review is to discuss the potential role of ENPP1 as a determinant of systemic insulin resistance and risk for its complications in humans.

The majority of animal studies to date have been consistent on the effects of ENPP1 impairing insulin stimulation of insulin receptor (IR) activation and downstream signaling. Early studies by Goldfine et al. [13] showed systemic insulin resistance in transgenic mice, overexpressing ENPP1 in liver and skeletal muscle. More recently, we [14] have created a transgenic mouse model (C57/Bl6 background) with targeted over-expression of human ENPP1 in adipocytes, using aP2 promoter in the transgene construct (Adipose-TG). After high fat diet with pair-feeding protocol, AdiposeENPP1-Tg and wild type mice had similar weight. However, AdiposeENPP1-Tg mice developed fatty liver in association with changes in adipose tissue, characterized by smaller adipocyte size, decreased phosphorylation of insulin receptor Tyr1361 $_{\text {and }} \mathrm{Akt}_{\text {Ser473. }}$. These changes in adipose tissue function and fat distribution were associated with systemic abnormalities of lipid and glucose metabolism, including increased plasma concentrations of fatty acid, triglyceride, plasma glucose and insulin during Intra-Peritoneal Glucose Tolerance Test (IPGTT) and impaired glucose suppression during Insulin Tolerance Test (ITT). Thus, over-expression of ENPP1 in an adipose tissue (AT)-selective transgenic model can play a role in adipose tissue dysfunction, with systemic consequences on glucose and lipid metabolism

${ }^{*}$ Corresponding author: Nicola Abate, M.D, Department of Medicine, Division of Endocrinology, Stark Diabetes Center, Institute for Translational Science (ITS), The University of Texas Medical Branch at Galveston, Texas, USA, Tel: 409-772-6314; Fax: 409-772-8709; E-mail: niabate@utmb.edu

Received: October 10, 2012 Accepted: October 12, 2012 Published: October 15, 2012 typically found in the metabolic syndrome: induced adipocyte insulin resistance, elevated plasma free fatty acid levels, ectopic fat distribution (fatty liver) and systemic insulin resistance.

A study by Zhou et al. [15] investigated the effect of suppression of ENPP1 expression and demonstrated that knockdown of ENPP1 with siRNA significantly increases insulin-stimulated Akt phosphorylation in $\mathrm{HuH7}$ human hepatoma cells. In vivo study of knockdown PC-1 expression in the liver of $\mathrm{db} / \mathrm{db}$ mice with ENPP1-1 short hairpin RNA adenovirus revealed $80 \%$ lower hepatic ENPP1 mRNA levels, and decreased fed and fasting plasma glucose with a concomitant improved oral glucose tolerance. Taken together, these results demonstrate that suppression of ENPP1 expression improves insulin sensitivity, supporting the hypothesis that ENPP1 inhibition is a potential therapeutic target for the treatment of type 2 diabetes.

Despite the existing evidence from animal studies, the effect of ENPP1 on complications of insulin resistance in humans remains controversial. Many previous studies showed positive association between the 121Q genetic variant and risk for type 2 diabetes and cardiovascular disease [4-12], whereas other studies have been negative [16]. The largest meta-analysis in type 2 diabetes to date [10] conducted on European populations showed a modest increase in risk for type 2 diabetes of the ENPP1 121Q allele. A similar meta-analysis in European adult population in 24,324 subjects [11], including 11,372 obese and 12,952 control subjects showed a statistically significant association between $121 \mathrm{Q}$ allele and obesity [OR $(95 \% \mathrm{CI})$ : 1.25 (1.04-1.52) $\mathrm{P}=0.021$ ] under a recessive model of inheritance (QQ vs. $\mathrm{KK}+\mathrm{KQ})$, without heterogeneity or publication bias. We have reported a significant interaction between ENPP1 121Q allele, age and BMI [17]. These interactions could explain some of the apparent discrepancies among genetic association studies conducted in various populations.

To better define the effect of ENPP1 in humans, we [18] recently conducted a study on the relationship of ENPP1 expression level and insulin resistance in 134 young normoglycemic volunteers. Body composition studies, hyperinsulinemic-euglycemic clamps and adipose tissue biopsy were obtained to test the overall hypothesis that ENPP1 over-expression contributes to AT dysfunction and systemic insulin resistance. Results showed a significant correlation between body fat content and insulin resistance for both males and females $(\mathrm{r}=-0.72 ; \mathrm{p}<0.0001$ and $\mathrm{r}=-0.60 ; \mathrm{p}<0.0001$, respectively), but there was a clear gender difference in the effects of ENPP1 expression on systemic insulin resistance. When compared to those who had low expression, the men but not the women with high ENPP1 expression had larger reductions in Rd-values during hyperinsulinemic clamps with increasing body fat content. These results clearly pointed out an interaction between ENPP1 expression and obesity, as well as an interaction between ENPP1 expression and gender. In both genders, ENPP1 over-expression was associated with up-regulation of genes involved in fatty acid metabolism and inflammation in AT $(\mathrm{p}<0.05$ for SREBP1c, DGAT, CD36, ASP, HSL, CD68, MAC1 and STAT3, adjusted for total or abdominal fat). Plasma fatty acid was higher in the high-ENPP1. Increased ENPP1 expression associates with AT dysfunction in both genders. These findings are concordant with the AdiposeENPP1-Tg phenotype and identify ENPP1 as playing a role in 
modulating obesity-related insulin resistance in humans. These data support the notion that ENPP1 should be considered both a marker and a novel potential target of therapy, to improve AT function and systemic glucose metabolism.

Recent study by Goldfine et al. [19] evaluated the metabolic impact of various common ENPP1 polymorphisms, including K121Q, rs1044498, and IVS20delT-11, rs1799774, and $A \rightarrow G+1044 T G A$, rs7754561. In 1112 subjects of non-Hispanic American white European ancestry, two of the ENPP1 variants-A $\rightarrow \mathrm{G}+1044 \mathrm{TGA}$ were significantly associated with hypertriglyceridemia (odds ratio $[\mathrm{OR}] 1.48,95 \%$ confidence interval $[\mathrm{CI}], 1.54-1.82, \mathrm{P}=0.002)$ and IVS20delT-11 (OR 1.41, 95\% [CI], 1.08-1.84, $\mathrm{P}=0.012$ ). Interestingly, this association was significant in the men but not women. Furthermore, the most widely studied variant, the K121Q was not found to associate with hypertriglyceridemia in any group or subgroup analysis. It is important to note that the hypertriglyceridemic subjects recruited in this study had BMI values in the overweight range, whereas the normolipidemic controls had lower BMI (28.7 vs. $25.1 \mathrm{~kg} / \mathrm{m}^{2}$ ). Both study groups were recruited from lipid clinic, which could have enriched the study population with metabolically abnormal subjects.

A prospective study of average duration of 37 months was conducted by Trischitta et al. [20] to evaluate the effects of ENPP1 $121 \mathrm{Q}$ variant on major cardiovascular events (myocardial infarction [MI], stroke and cardiovascular death). The study was conducted in the population of the Gargano Heart Study (GHS; $n=330$ with type 2 diabetes and coronary artery disease), the Tor Vergata Atherosclerosis Study (TVAS; $\mathrm{n}=141$, who had MI), and the Cardiovascular Risk Extended Evaluation in Dialysis (CREED) database ( $\mathrm{n}=266$ with end-stage renal disease). Results showed incidence of cardiovascular events per 100 person-years was 4.2 in GHS, 10.8 in TVAS and 11.7 in CREED. Hazard ratios (HRs) for KQ+QQ versus individuals carrying the K121/K121 genotype (KK) individuals were 1.47 (95\% CI, 0.80-2.70) in GHS, 2.31 (95\% CI, 1.22-4.34) in TVAS, 1.36 (95\% CI, 0.88-2.10) in CREED, and 1.56 (95\% CI, 1.15-2.12) in the three cohorts combined. In the 395 diabetic patients, the Q121 variant predicted cardiovascular events among obese, but not among nonobese individuals (HR 5.94 vs. $0.62, \mathrm{P}=0.003$ for interaction). A similar synergism was observed in cross-sectional studies, with age at MI being 3 years younger in Q121 carriers than in KK homozygotes among obese, but not among non-obese patients $(\mathrm{P}=0.035$ for interaction). An additional important result of this study is that the effect of the Q121 variant was modulated by obesity in diabetic patients, among whom the risk of incident events was five times higher in Q121 than in KK genotype carriers. The Q121variant seems to eliminate the paradoxical protective effect (a whole tend to have a lower risk of future cardiovascular events) of obesity.

Another recent study with weight loss intervention of 211 nondiabetic overweight-obese individuals revealed that the amelioration of glucose homeostasis observed after weight loss is modulated by the ENPP1 K121Q polymorphism [21]. Body mass index (BMI), fasting glucose, homeostasis model assessment of insulin resistance (HOMA-IR index) and lipid levels were obtained before and after 6-week lifestyle intervention (LI; diet and exercise), and their changes calculated as baseline minus 6-week values. LI decreased BMI, glucose, HOMA-IR and triglyceride levels ( $\mathrm{p}<0.001$ for all). No difference across genotype groups (160 KK and $51 \mathrm{KQ}$ or QQ-named as XQindividuals) was observed in these changes. In a multivariate model, BMI changes predicted fasting glucose changes $(\beta=0.139 \mathrm{mmol} / \mathrm{L}$
( $2.50 \mathrm{mg} / \mathrm{dl})$ for 1 unit BMI change, $\mathrm{p}=0.005)$. This correlation was not significant among KK individuals $(\beta=0.082 ; p=0.15)$, while much steeper and highly significant among XQ individuals $(\beta=0.336$; $\mathrm{p}=0.00008$ ) ( $\mathrm{p}$-value for $\mathrm{Q} 121$-by-weight loss interaction=0.047). This study thus, confirms that individuals carrying the ENPP1 Q121 variant are highly responsive to the effect of weight loss on fasting glucose; and reinforces the previously suggested hypothesis that the Q121 variant interacts with adiposity in modulating glucose homeostasis.

In summary, the common observation in the human studies discussed above is that the inhibitor effect of ENPP1 on insulin signaling has been replicated in subjects with higher expression level of the ENPP1, in the presence of $121 \mathrm{Q}$ gene variant, obesity, type 2 diabetes or significant lipid disorder, which may be recapitulating the extreme metabolic condition in animal study. In animal models, target gene expression level was manipulated by overexpression or knockdown, in order to achieve biological effect. Extreme metabolic challenge such as high diet is often applied to magnify the effect in animal study. As the opposite, studies conducted in general human population may fail to reveal the linkage between ENPP1 and insulin resistance due to confounding factors, such as heterogeneous ethnic origin and environmental background. Larger meta-analysis may be needed to overcome the influence of heterogeneity.

Overall, the ENPP1 K121Q polymorphism and other variants is an independent risk factor of insulin resistance and metabolic syndrome. However, we have described a significant interaction between the gene and BMI [17]. We have also described a gender difference in the association between ENPP1 expression and insulin resistance [18]. Therefore, the degree of functional impairment determined by the $121 \mathrm{Q}$ variant may not be sufficient to determine a measurable phenotype, unless interaction variables are taken into account. Synergistic effect is observed with increased cellular expression of the gene or additional metabolic stress such as over-nutrition, obesity, type 2 diabetes and hypertriglyceridemia. Further more, more dramatic improvement on glucose homeostasis is observed in ENPP1 K121Q variant with weight reduction, suggesting that this group of people may benefit more from life style intervention, alleviating metabolic challenge induced by positive caloric balance.

\section{Acknowledgements}

We acknowledge support by NIH grant R01 DK072158 from NIDDK; by CTSA-NIH UL1RR029876 from the NCRR and Shriners' Burn Hospital Grant 71007.

\section{References}

1. Maddux BA, Goldfine ID (2000) Membrane glycoprotein PC-1 inhibition of insulin receptor function occurs via direct interaction with the receptor alphasubunit. Diabetes 49: 13-19.

2. Liang J, Fu M, Ciociola E, Chandalia M, Abate N (2007) Role of ENPP1 on adipocyte maturation. PLoS One 2: e882.

3. Bacci S, Ludovico O, Prudente S, Zhang YY, Di Paola R, et al. (2005) The $\mathrm{K} 121 \mathrm{Q}$ polymorphism of the ENPP1/PC-1 gene is associated with insulin resistance/atherogenic phenotypes, including earlier onset of type 2 diabetes and myocardial infarction. Diabetes 54: 3021-3025.

4. Frittitta L, Baratta R, Spampinato D, Di Paola R, Pizzuti A, et al. (2001) The Q121 PC-1 variant and obesity have additive and independent effects in causing insulin resistance. J Clin Endocrinol Metab 86: 5888-5891.

5. Kubaszek A, Pihlajamaki J, Karhapaa P, Vauhkonen I, Laakso M (2003) The $\mathrm{K} 121 \mathrm{Q}$ polymorphism of the $\mathrm{PC}-1$ gene is associated with insulin resistance but not with dyslipidemia. Diabetes Care 26: 464-467. 
6. Abate N, Carulli L, Cabo-Chan A Jr, Chandalia M, Snell PG, et al. (2003) Genetic polymorphism PC-1 K121Q and ethnic susceptibility to insulin resistance. J Clin Endocrinol Metab 88: 5927-5934

7. Hamaguchi K, Terao H, Kusuda Y, Yamashita T, Hazoury Bahles JA, et al (2004) The PC-1 Q121 allele is exceptionally prevalent in the Dominican Republic and is associated with type 2 diabetes. J Clin Endocrinol Metab 89 1359-1364.

8. Abate N, Chandalia M, Satija P, Adams-Huet B, Grundy SM, et al. (2005) ENPP1/PC-1 K121Q polymorphism and genetic susceptibility to type 2 diabetes. Diabetes 54: 1207-1213.

9. Meyre D, Bouatia-Naji N, Tounian A, Samson C, Lecoeur C, et al. (2005) Variants of ENPP1 are associated with childhood and adult obesity and increase the risk of glucose intolerance and type 2 diabetes. Nat Genet 37 : 863-867.

10. McAteer JB, Prudente S, Bacci S, Lyon HN, Hirschhorn JN, et al. (2008) The ENPP1 $\mathrm{K} 121 \mathrm{Q}$ polymorphism is associated with type 2 diabetes in European populations: evidence from an updated meta-analysis in 42,042 subjects. Diabetes 57: 1125-1130.

11. Wang R, Zhou D, Xi B, Ge X, Zhu P, et al. (2011) ENPP1/PC-1 gene K121Q polymorphism is associated with obesity in European adult populations: evidence from a meta-analysis involving 24,324 subjects. Biomed Environ Sci 24: $200-206$

12. Szuszkiewicz M, Bell J, Vazquez M, Adams-Huet B, Grundy SM, et al. (2011) ENPP1/PC-1 K121Q and other predictors of posttransplant diabetes. Metab Syndr Relat Disord 9: 25-29.

13. Maddux BA, Chang YN, Accili D, McGuinness OP, Goldfine ID, et al. (2006) Overexpression of the insulin receptor inhibitor PC-1/ENPP1 induces insulin resistance and hyperglycemia. Am J Physiol Endocrinol Metab 290: E746-E749.
14. Pan W, Ciociola E, Saraf M, Tumurbaatar B, Abate N, et al. (2011) Metabolic consequences of ENPP1 overexpression in adipose tissue. Am J Physiol Endocrinol Metab 301: E901-E911.

15. Zhou HH, Chin CN, Wu M, Ni W, Quan S, et al. (2009) Suppression of PC-1/ ENPP-1 expression improves insulin sensitivity in vitro and in vivo. Eur $J$ Pharmacol 616: 346-352

16. Rasmussen SK, Urhammer SA, Pizzuti A, Echwald SM, Ekstrom CT, et al (2000) The K121Q variant of the human PC-1 gene is not associated with insulin resistance or type 2 diabetes among Danish Caucasians. Diabetes 49 : 1608-1611.

17. Chandalia M, Grundy SM, Adams-Huet B, Abate N (2007) Ethnic differences in the frequency of ENPP1/PC1 $121 \mathrm{Q}$ genetic variant in the Dallas Heart Study cohort. J Diabetes Complications 21: 143-148.

18. Chandalia M, Davila H, Pan W, Szuszkiewicz M, Abate N, et al. (2012) Adipose Tissue Dysfunction in Humans: A Potential Role for the Transmembrane Protein ENPP1. J Clin Endocrinol Metab.

19. Tanyolaç S, Bremer AA, Hodoglugil U, Movsesyan I, Goldfine ID, et al. (2009) Genetic variants of the ENPP1/PC-1 gene are associated with hypertriglyceridemia in male subjects. Metab Syndr Relat Disord 7: 543-548.

20. Bacci S, Rizza S, Prudente S, Spoto B, Trischitta V, et al. (2011) The ENPP1 Q121 variant predicts major cardiovascular events in high-risk individuals: evidence for interaction with obesity in diabetic patients. Diabetes 60: 10001007.

21. Maranghi M, Prudente S, D’Erasmo L, Morini E, Ciociola E, et al. (2012) The ectonucleotide pyrophosphatase phosphodiesterase 1 (ENPP1) K121Q polymorphism modulates the beneficial effect of weight loss on fasting glucose in non-diabetic individuals. Nutr Metab Cardiovasc Dis.

\section{Author Affiliation}

'Department of Medicine, Division of Endocrinology, Stark Diabetes Center, Institute for Translational Science (ITS), The University of Texas Medical Branch at Galveston, Texas, USA

\footnotetext{
submissions

* 50 Journals

* 21 Day rapid review process

* 1000 Editorial tean

2 Million readers

* More than 5000 lacentage

* Publication immediately after acceptance

* Quality and quick editorial, review processing
}

Submit your next manuscript and get advantages of SciTechnol

Submit your next manuscript at • www.scitechnol.com/submission 